\title{
Primary frequency control in a power system with battery energy storage systems
}

\author{
Mihai Sanduleac, Lucian Toma, Mircea Eremia, Valentin A. Boicea, Dorian Sidea, Alexandru Mandis \\ Department of Electrical Power Systems, University Politehnica of Bucharest, Romania
}

\begin{abstract}
This paper addresses the feasibility of a battery energy storage system (BESS) contribution to primary frequency control by simulating its state of charge over several days and by using frequency measurements in the Romanian power system.

A BESS correction algorithm has been developed to overcome the average frequency asymmetry which may bring the state of charge to zero or $100 \%$, thus not allowing further primary frequency control due to total discharge or total charge of the storage resource.

It is demonstrated that for a number of selected days the algorithm provides good results, the primary frequency control is delivered over entire days, and that a reserve of energy remains in the battery for eventual disturbances in the system, for both over and under frequency needs.
\end{abstract}

Keywords-BESS, State of charge, primary frequency control.

\section{INTRODUCTION}

The increasing share of generation from renewable energy sources (RES) in power systems comes with various challenges, such as less predictable generation variation, especially due to wind intermittency, voltage stability issues, power unbalance, and frequency variations. One solution to overcome these problems is the use of battery energy storage systems (BESS). Basically, their role is to charge (consume energy) when there is generation surplus, and to inject power back into the grid during periods without generation from RES. In the same train of thought, one must mention that the frequency variation within the power system is caused by delayed correlation between power generation and load [1]. Thereby, the BESS can become an attractive solution when it comes to frequency regulation over short periods of time (of maximum 15 minutes), which is specific to the primary frequency control (PFC). In $[2,3]$ the PFC has been identified as the most important application of the BESS.

Another important aspect related to this topic is the economic one. The annual profit resulted from the use of BESS for the PFC is estimated between US\$236-US\$439 per $\mathrm{kW}$, on the US electricity market $[2,4]$. Practically, the BESS employed for the PFC can be used not only in the microgrids but also in interconnected power systems, as will be described in the sequel.

The PFC dates back to 1936 and was introduced by Georges Darrieus in collaboration with Jean-Gustave Fallou
[1,5]. Initially, it was called autonomy or Darrieus condition [5]. According to the basic principle, in case of interconnected power systems, when the frequency deviation occurs, the sources from all the power systems must contribute in order to overcome the disturbance, independently from each other (solidarity principle). This participation takes the form of a variation in the power exchanges between the systems [5].

In Romania, for instance, the primary frequency reserve must be fully deployed within 30 seconds after disturbance occurrence, and must be maintained for at least 15 minutes. The purpose of the PFC is to stabilize the frequency about a quasi-steady-state value. In order to restore the frequency back to the reference value, the secondary frequency control loop is employed; the secondary frequency control (called also automatic frequency restoration) reserve must be activated within 30 seconds after the perturbation occurrence and should be fully available within 15 minutes and maintained for unlimited period of time. Optimal unit commitment for frequency control is performed within the tertiary level, called also manual frequency restoration $[6,7]$.

In the ENTSO-E network of the Continental Europe, the primary frequency control is activated if frequency falls outside the interval $49.95-50.05 \mathrm{~Hz}$ [6]. The power can be linearly increased or decreased according to a droop coefficient. The possibility to ramp up or down the power is given by the fact that the generating units are always operated at a lower point with respect to their maximum capacity.

In order to evaluate the performance of a BESS, one has to know the Power/Energy ratio (P/E). In case of frequency regulation, high power capacity over few minutes is necessary, which suggests that $\mathrm{P} / \mathrm{E}$ should be high [8]. Other key factor related to the BESS sizing is the average current rating. This parameter, together with the depth of discharge (DoD) or the duty cycle, plays an important role also when it comes to cycle life. The degradation of a BESS is equated with an increased internal resistance and reduced capacity [8].

On the other hand, the BESS cycle life is always finite and strongly influenced by the operating conditions. At the same time, if a battery had up to a certain moment 800 cycles at DoD $100 \%$, this means that it is still capable of having more than 800 cycles at a lower DoD [8].

Various BESS technologies have been used for PFC. These include: Lead Acid, Sodium Metal Halide, Sodium Sulfur or Li-ion. The characteristics of each of these technologies are presented in Table 1 [6].

This work has been funded by the European Commission through the Horizon 2020 research and innovation programme, under the RESERVE and Storage4Grid project grant agreements No. 727481 and 731155 , as well as by the Romania UEFISCDI PN-III-CERC-CO-PTE-2016 program under grant agreement no. 22PTE/2016. 
Table 1. Various BESS technologies used in PFC.

\begin{tabular}{|c|c|c|c|c|}
\hline & Lead Acid & $\begin{array}{c}\text { Sodium } \\
\text { Metal } \\
\text { Halide }\end{array}$ & $\begin{array}{c}\text { Sodium } \\
\text { Sulfur }\end{array}$ & Li-ion \\
\hline $\begin{array}{c}\text { Response } \\
\text { time [ms] }\end{array}$ & 100 & 100 & 100 & 100 \\
\hline $\begin{array}{c}\text { Storage } \\
\text { period }\end{array}$ & $\begin{array}{c}15 \mathrm{~min}- \\
3 \mathrm{hr}\end{array}$ & $\begin{array}{c}15 \mathrm{~min}- \\
3 \mathrm{hr}\end{array}$ & $4-6 \mathrm{hr}$ & $\begin{array}{c}15 \mathrm{~min} \\
-1 \mathrm{hr}\end{array}$ \\
\hline $\begin{array}{c}\text { Efficiency } \\
{[\%]}\end{array}$ & $\sim 85 \%$ & $\sim 85 \%$ & $\sim 70 \%$ & $\sim 90 \%$ \\
\hline
\end{tabular}

Another general aspect which has to be taken into account is that the frequency should be regulated using the cheapest energy. This would be another reason which makes the BESS an attractive solution when it comes to PFC.

\section{BESS CONTRIBUTION TO PRIMARY FREQUENCY CONTROL}

The frequency deviation in a power system, at a certain time instant $t$, is simply calculated as

$$
\Delta f(t)=f_{\text {ref }}-f_{\text {real }}(t)
$$

where and $f_{\text {ref }}$ is the reference value, i.e. $50 \mathrm{~Hz}$ in Europe, and $f_{\text {real }}(\mathrm{t})$ is the metered frequency at the time instant $t$.

The participation power of the BESS required to restore the frequency at the time instant $t$ is

$$
\Delta P_{b a t}(t)=-\Delta f(t) \frac{P_{\max , b a t}}{\Delta f_{\max }}
$$

where $P_{\max , b a t}$ is the maximum rating power of the battery, and $\Delta f_{\max }$ is the maximum frequency deviation within which the battery will respond, thus resulting in a participation factor $P_{\max , b a t} / \Delta f_{\max }[\mathrm{MW} / \mathrm{Hz}]$

The total energy available in the battery is calculated at every time instant $t$ (the simulation time step is 1 second) by [9]:

$$
E_{b a t}(t)=E_{b a t}(t-1)+\frac{\Delta P_{b a t}(t)}{3600}
$$

When the battery state of charge (SoC) decreases below a threshold (e.g. 20 to $25 \%$ ) or is above a similar threshold (e.g. 75 to $80 \%$ ), an additional charging/discharging power of constant value $P_{l t c h}$ (lt_ch denotes the long term charging/discharging) is added to the power required by the primary frequency control, expressed in (4), i.e.:

$$
\Delta P_{b a t}(t)=-\Delta f(t) \frac{P_{\max , b a t}}{\Delta f_{\max }} \pm P_{l t_{-} c h}(t)
$$

\section{BESS SIMULATIONS BASED ON FREQUENCY METERED DATA}

The goal of this paper is to present a PFC carried out with a BESS based on data from the continental European power system for one day time frame.

\section{A. Scenario 1: Test Day 1}

Fig. 1 shows the frequency variation over 24 hours, based on measurements done at a point in Romania (synchronized with the ENTOS-E), with $10 \mathrm{mHz}$ sampling rate, and 1 second averaging time interval. Let us denote this set of data by Test Day 1, or TD1.

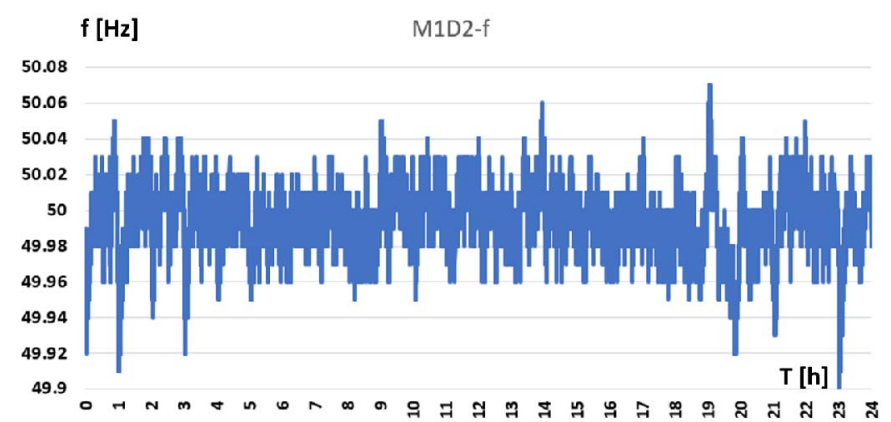

Fig. 1. Frequency evolution over one day (TD1) in Romania.

From Fig. 1, it can be seen that the frequency variation is in the range between 49.9 and $50.07 \mathrm{~Hz}$. The average value for the entire day was $49.9947 \mathrm{~Hz}$, which shows a small deviation from the nominal frequency.

We consider using a $2 \mathrm{MWh}$ BESS, of +/-10 MW rated output power. The maximum power is engaged up to a frequency deviation of $500 \mathrm{mHz}$.

By applying the previous formulae, we can get the BESS state of charge (SoC) over the whole day, as shown in Fig. 2, considering that at the beginning of the day the battery is half charged $(2 \mathrm{MWh} \times 50 \%=1 \mathrm{MWh})$.

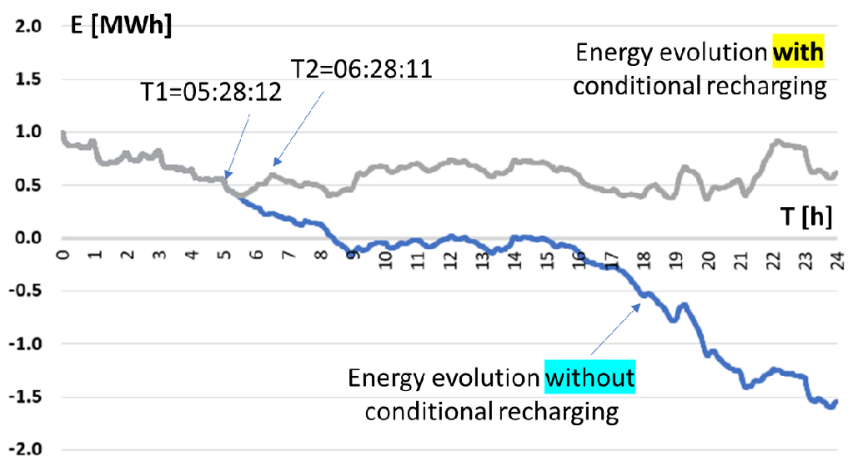

Fig. 2. BESS state of charge evolution over DT1, with/without charging compensation.

Figure 2 shows that without compensation, in this particular day the battery is completely discharged after around 8 hours because the average frequency was smaller than the nominal value. The blue line shows that, as a need for primary frequency control, in the last part of the day the SOC is mostly negative, which is not physically possible (BESS can discharge until zero only). For a charging compensation if SoC reaches a value below $20 \%$, the SOC remains in the control region over the whole day.

The charging function is triggered when SoC falls bellow $20 \%$. The charging power $P_{l t_{-} c h}$ has been chosen to be only $5.8 \%$ of the total power and maintained constant for an entire 
hour after activation. In these conditions the battery is seen as a load, not as a frequency control entity. The charging orders of the BESS over the whole day are presented in Fig. 3.

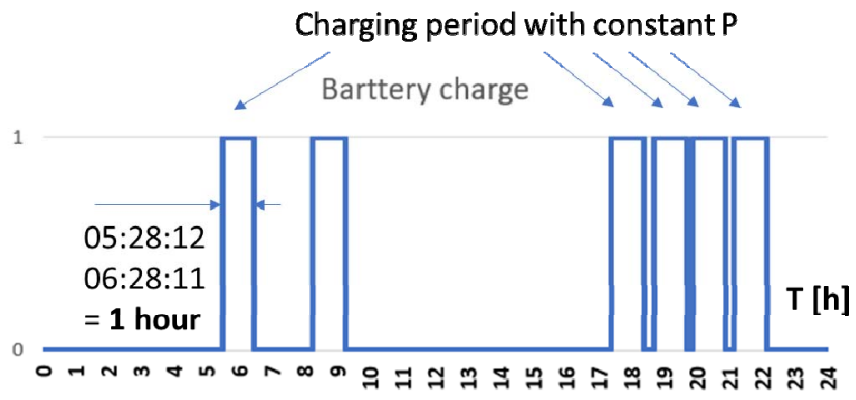

Fig. 3. BESS state of charge charging orders for DT1.

\section{B. Scenario 2: Test Day 2}

In order to prove the robustness of the algorithm, the same procedure should be applied for several days of recorded data. Let us now consider in this scenario a new set of data, denoted by TD2.

Fig. 4 shows a daily evolution where the BESS reaches in the same day both low and high SoC, the study being made for the $25 \%$ and $75 \%$ thresholds that trigger the charging or discharging commands. The figure illustrates the evolution of $\mathrm{SoC}$ with and without correction and shows that, in this particular day, the BESS behaves satisfactorily even without the correction algorithm. However, with correction the band of $\mathrm{SoC}$ variation over the entire day is reduced, bringing more reserve for unexpected disturbances. The average value of the frequency for the entire day TD2 was $49.9993 \mathrm{~Hz}$, which is very near to the nominal value of $50 \mathrm{~Hz}$.

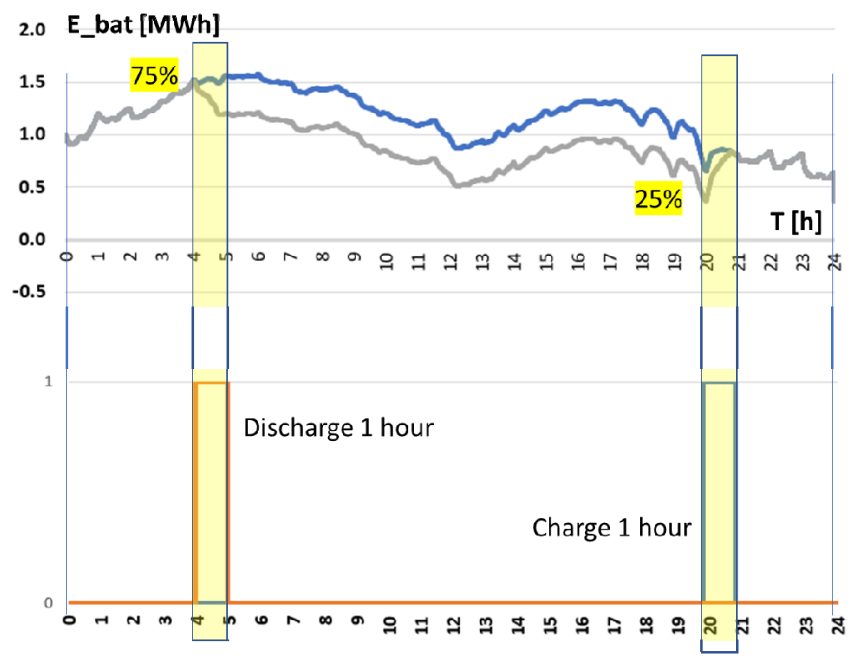

Fig. 4. BESS charging status for a day (TD2) with both charge and discharge orders.

\section{Scenario 3: Test Day 3}

For diversity let us consider one more day, denoted by TD3. Fig. 5 shows the SoC for this specific day. One can see that the corrections are strictly necessary, as the normal evolution of the BESS energy would be completely depleted after approx. 7 hours, while the trend which continues the whole day. The average value for the entire day was 49.9929 $\mathrm{Hz}$, only slightly lower than in the first studied case and the frequency evolution required for 6 charging periods in order to keep SoC in the boundaries.

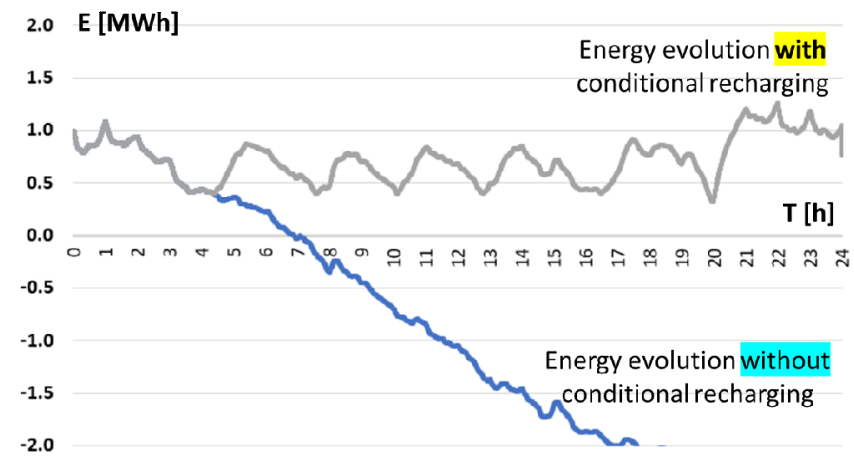

Fig. 5. BESS state of charge evolution over the day TD3, with and without charging compensation.

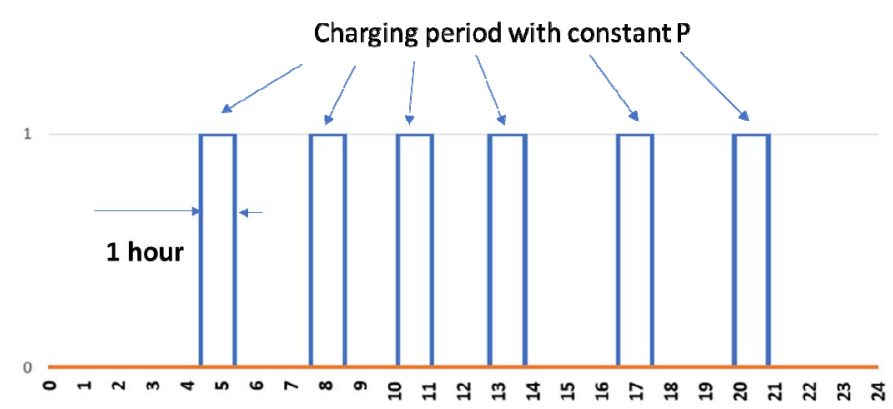

Fig. 6. BESS state of charge charging orders for the day TD3.

\section{Comparing the results}

Fig. 7 shows the BESS SoC evolution for five different days, including those presented above. One day only does not need the corrections algorithm (see Fig. 4).

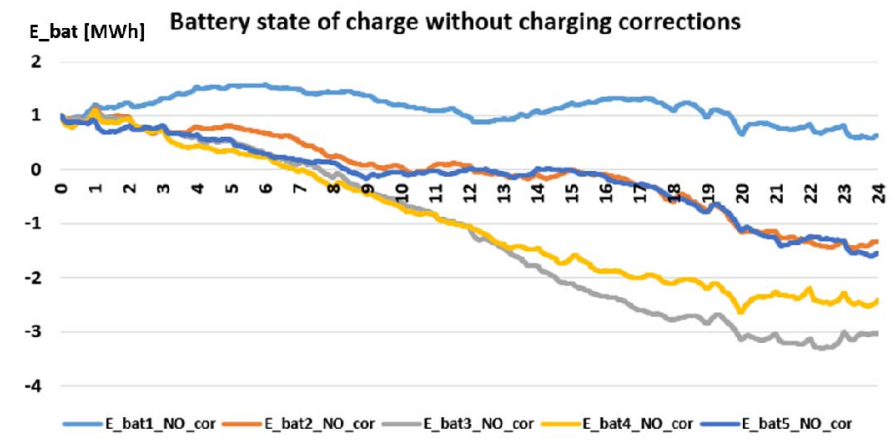

Fig. 7. BESS state of charge evolution in five different days, without charging compensation.

Fig. 8 shows the same BESS SoC, but with applied correction algorithm for charging or discharging the battery based on the presented triggers. In all situations the $\mathrm{SoC}$ remains within a certain range, while a reserve of around $25 \%$ of BESS energy for both frequency disturbance directions (over or under frequency) is maintained. 
2

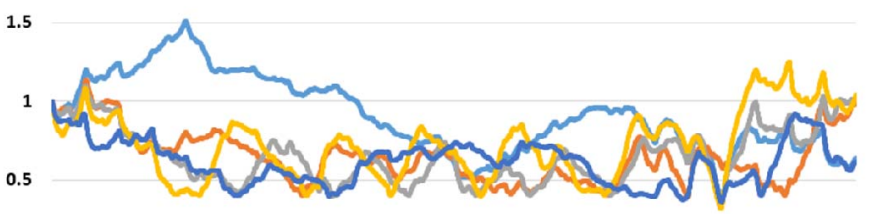

$\mathrm{T}[\mathrm{h}]$

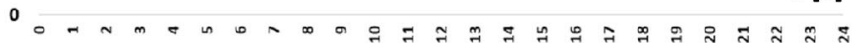

-E_bat1_COR - E_bat_COR - E_bat3_COR - E_bat4_COR - E_bat5_COR

Fig. 8. BESS state of charge evolution in five different days, with charging compensation.

\section{INFLUENCE OF LONG-TERM CHARGING POWER $P_{L T_{C} C H}$}

In above studied cases the charging power $P_{l t}$ ch has been set to $5.8 \%$ of the BESS inverter rating, which is in our case $10 \mathrm{MW}$. This section analyses the influence of the long-term charging power $P_{l t}$ ch on the charging/discharging schedule. In order to assess the influence of this factor, one day has been selected only, respectively TD3.

The following values for the long-term charging power $P_{l+c h}$ have been selected: $4.0 \%, 5.5 \%, 8.0 \%$ and $9.5 \%$, with the second case having a value nearby to the TD3 situation already presented.

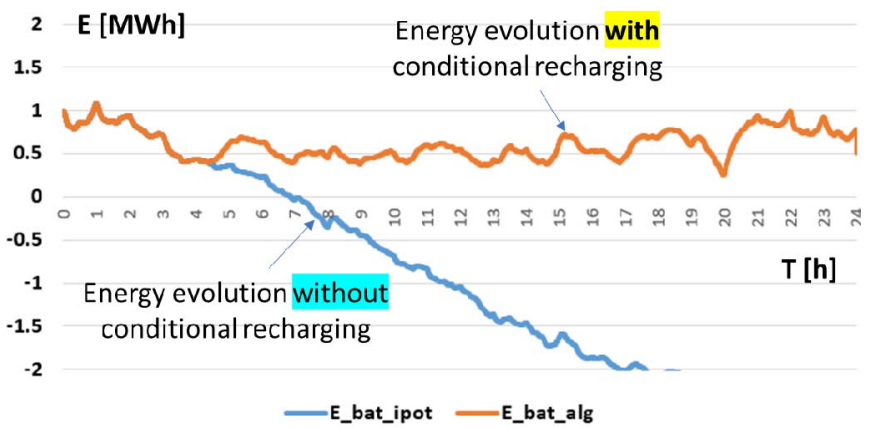

Fig. 9. BESS state of charge evolution over the day TD3, with and without charging compensation, for $P_{l t c h}=4.0 \%$.

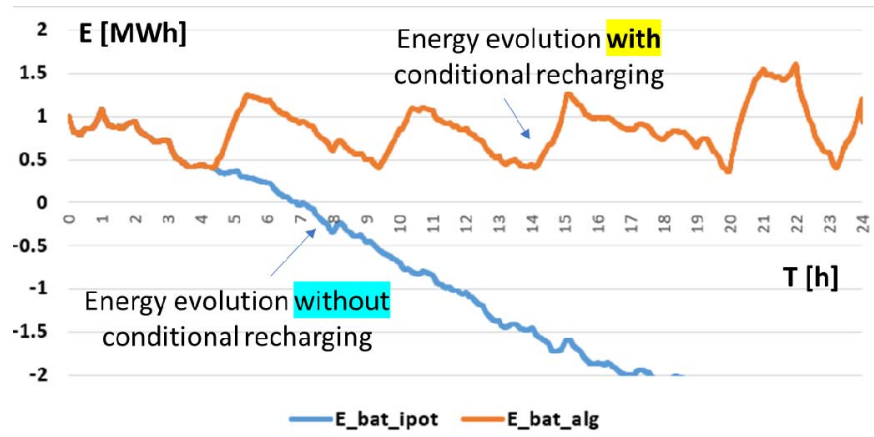

Fig. 10. BESS state of charge evolution over the day DT3, with and without charging compensation, for $P_{l t c h}=9.5 \%$.

Fig. 9 shows the energy variation for $P_{l t} c h=4.0 \%$, and Fig. 10 illustrates the energy variation for $P_{l t_{-} c h}=9.5 \%$ (the lower and upper selected values). The low power for longterm charging or discharging makes the conditional recharging more stable around $\mathrm{SoC}=50 \%$ while the high value of $9.5 \%$ of the BESS inverter rating results in a higher variation around the middle value, suggesting that the $P_{l+}$ ch value is too high.

Figures 11 through 14 show the battery charge or discharge trigger signal for various values of the charging power $P_{l t \_c h}$.

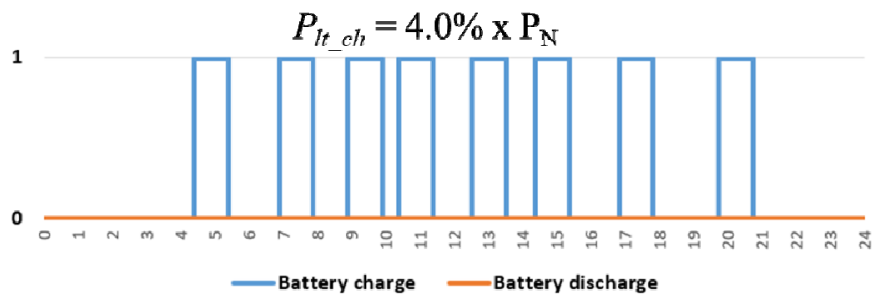

Fig. 11. BESS state of charge charging orders for TD3 for $P_{l t_{-} c h}=4.0 \%$.

$$
P_{I t_{-} c h}=6.0 \% \times \mathrm{P}_{\mathrm{N}}
$$

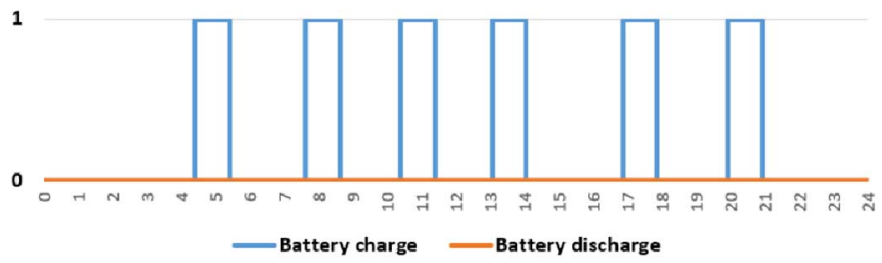

Fig. 12. BESS state of charge charging orders for TD3 for $P_{l+c h}=6.0 \%$.

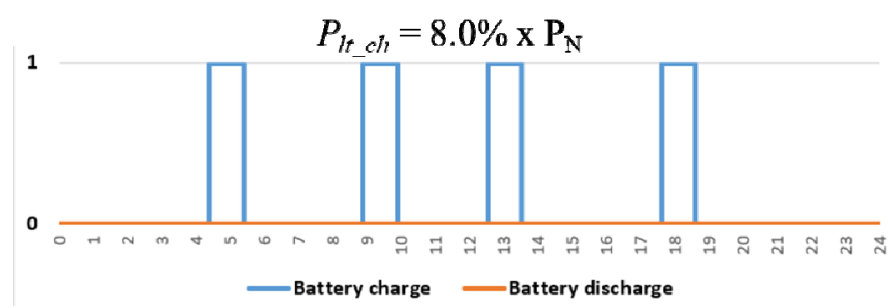

Fig. 13. BESS state of charge charging orders for TD3 for $P_{l t_{-} c h}=8.0 \%$.

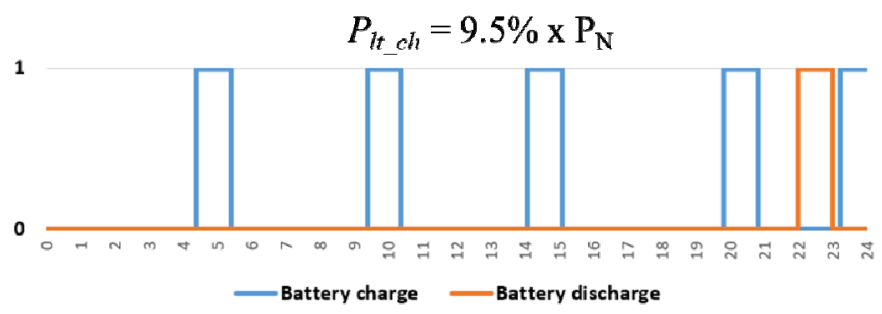

Fig. 14. BESS state of charge charging orders for TD3 for $P_{l t_{-} c h}=9.5 \%$.

The above figures show that the long-term charging power $P_{l t c h}$ does not need to be too high, as such situations could bring unwanted cycles of charging and discharging. This is observed in Fig. $14\left(P_{l t_{-} c h}=9.5 \%\right)$, where there is an unnecessary cycle of discharging at the end of the day, due to aggressive charging in the previous period. At the same time, a too small charging power $P_{l t_{c} c h}$ brings more charging cycles in the studied period. For $P_{l t_{-} c h}=4.0 \% 8$ charging cycles are needed, for $P_{l t c h}=6.0 \% 6$ cycles are needed, whereas 4 cycles only are needed for $8 \%$. For values higher than $9.5 \%$ the 
complementary charging and discharging activities are also present, and the time for the cycle need to be reduced below one hour due to overlapping, which creates an even less desired situation, with frequent "oscillations" on the BESS operating regime.

This assessment suggests that for the frequency pattern already studied, an $P_{l t \_c h}$ of $8 \%$ would be the best solution for a reduced number of cycles.

\section{CONCLUSIONS}

This paper demonstrates the feasibility of a BESS system for primary frequency control by simulating its state of charge over several days and by using real measurements of frequency in the ENTSO-E system.

An algorithm for superposing long-term charging and discharging cycles (one hour long in our simulations) proved to ensure BESS in healthy limits, while the constant charging/discharging can be perceived at system level as an unbalance to be addressed by the secondary frequency control level. It is demonstrated that for the selected days the algorithm works well, and that BESS is appropriate for primary frequency control even in non-symmetric evolution of the frequency over long periods of time, such as a whole day.

By simulations performed we found out that the long-term charging power $P_{l t \_c}$ can influence the number of charging cycles. Lower values of $P_{l t}$ ch has reduced the number of daily cycles down to $\mathrm{N}=4$, while higher value of $P_{l+c h}$, e.g. $8 \%$ in our case, a limit that avoid oscillations, has resulted in higher number of daily cycles. The analysis suggests that such simulations, performed on a large number of days with real frequency measurements, can result in an optimized $P_{l t c h}$ value for real situations. The same assessment shows that a too high value of $P_{l t c h}$ is not desirable, as bringing unnecessary complementary cycles of charging and discharging, which can stress the BESS.

\section{REFERENCES}

[1] S. A. Amamra, B. Francois and J. Lugaro, "Day-ahead primary power reserve planning and day-D primary frequency control of a Li-ion battery," 2015 IEEE Eindhoven PowerTech, Eindhoven, 2015, pp. 1-5.

[2] Y. J. Zhang, C. Zhao, W. Tang and S. H. Low, "Profit Maximizing Planning and Control of Battery Energy Storage Systems for Primary Frequency Control," in IEEE Transactions on Smart Grid, vol. 9, no. 2, pp. 712-723, March 2018.

[3] A. Oudalov, D. Chartouni, C. Ohler and G. Linhofer, "Value Analysis of Battery Energy Storage Applications in Power Systems," 2006 IEEE PES Power Systems Conference and Exposition, Atlanta, GA, 2006, pp. 22062211.

[4] P. Denholm, E. Ela, B. Kirby, and M. Milligan, "The role of energy storage with renewable electricity generation," Technical Report, National Renewable Energy Laboratory, 2010.

[5] E. Mariani and S.S. Murthy, Control of Modern Integrated Power Systems, London, UK: Springer Verlag, 1997.

[6] ENTSO-E, Network Code on Load-Frequency Control and Reserves, 28 June 2013.

[7] M. Eremia and M. Shahidehpour, (Editors), Handbook of Electrical Power System Dynamics: Modeling, Stability, and Control, Wiley \& IEEE Press, Power Engineering Series, Hoboken-USA, March 2013.

[8] M. Chawla, R. Naik, R. Burra and H. Wiegman, "Utility energy storage life degradation estimation method," 2010 IEEE Conference on Innovative Technologies for an Efficient and Reliable Electricity Supply, Waltham, MA, 2010, pp. 302-308.

[9] L. Toma, I. Tristiu, C. Bulac, A.-G. Neagoe-Ştefana, "Optimal generation scheduling strategy in a microgrid", IEEE Transportation Electrification Conference and Expo Asia-Pacific (ITEC-BUSAN 2016), Busan, South Korea, 1-4 June 2016. 